Published as: Stal, C., De Wulf, A., De Coene, K., De Maeyer, P., Nuttens, T. \& Ongena, T. (2012), Digital representation of Historical Globes: Methods to Make 3D and Pseudo-3D Models of 16th Century Mercator Globes. Cartographic Journal vol. 49 (2), 107-117.

\title{
Digital Representation of Historical Globes: Methods to Make 3D and Pseudo-3D Models of 16th Century Mercator Globes
}

\author{
Cornelis Stal, Alain De Wulf, Karen De Coene, Philippe De Maeyer, Timothy Nuttens, Thérèse Ongena \\ Department of Geography, Ghent University, Krijgslaan 281-S8, Ghent, Belgium
}

\begin{abstract}
In this paper, the construction of digital representations of a terrestrial and celestial globe will be discussed. Virtual digital (3D) models play an important role in recent research and publications on cultural heritage. The globes discussed in this paper were made by Gerardus Mercator $(1512-1594)$ in 1541 and 1551. Four techniques for the digital representation are discussed and analysed, all using high resolution photographs of the globes. These photographs were taken under studio conditions in order to get equal lighting and to avoid unwanted light spots. These lighting conditions are important, since the globes have a highly reflective varnish covering. Processing these images using structure from motion, georeferencing of separate scenes and the combination of the photographs with terrestrial laser scanning data results in true 3D representations of the globes. Besides, pseudo-3D models of these globes were generated using dynamic imaging, which is an extensively used technique for visualisations over the Internet. The four techniques and the consequent results are compared on geometric and radiometric quality, with a special focus on their usefulness for distribution and visualisation during an exhibition in honour of the $500^{\text {th }}$ birthday of Gerardus Mercator.
\end{abstract}

Keywords: Globes, Mercator, 3D modelling, virtual reality, comparison

\section{Introduction}

In 2012, the celebration of the $500^{\text {th }}$ birthday of Gerardus Mercator will take place. In honour of this special occasion, 3D models were generated of two of his famous globes for visualisation purposes. It concerns a terrestrial globe, constructed in 1541, and a celestial globe of 1551. Both globes are on display at the Mercator Museum in the city of Sint-Niklaas near Antwerp, Belgium.

The importance of virtual representations of globes has been discussed by different authors, such as Adami (2009), Gede (2009 a, b) or Hruby et al. (2005, 2006). These authors mainly focus on the data acquisition of the virtual representations of the globes. Adami (2009) discusses the use of 3D scanners or range cameras in order to generate triangulated models of globes that can be used to investigate the real shape of the sphere. Based on his work, it becomes clear that the use of 3D scanners is suitable for deformation measurements and supplements the research on the internal structure of the globe. Furthermore, he analyses the projective representation of the map content by using specialised software. Image-based representations of historical globes by projecting image parts on a sphere are also discussed (Dorffner, 1996, Hruby et al., 2006), as well as possible digital formats and viewers for these globes (Gede, 2009a, b; Hruby et al., 2005). An earlier example of such image based 
visualisation can be found on www.hcl.harvard.edu. Hruby et al. (2005, 2006) combine coloured images of the globe with black and white scans of the reprinted gores, in order to improve the readability of the textures. For the virtual representations of the globes used in this article, only the two globes were used as data source. The research of these authors will be used for a comparative study in this paper, complemented with the structure from motion modelling technique and dynamic imaging. The focus of this paper is on the 3D representation of globes for visualisation purposes, using the different implementations of the authors mentioned above.

After a brief presentation of the globes in section 2, the acquisition of the photographs in terms of studio configuration, camera configuration and image pre-processing is the topic of section 3 . Four modelling and visualisation techniques are discussed: dynamic images for the pseudo-3D model (paragraph 4.1), laser scanning and image draping (paragraph 4.2), structure from motion (paragraph 4.3) and georeferencing and merging fragments of the globe (paragraph 4.4). Section 5 considers the use of different software packages to visualise 3D models. Digital images of the globe were used for all modelling techniques. While the dynamic images do not result in a 3D-model, the others do. In the case of laser scanning and image draping the images are supplemented with a point set, generated by a 3D laser scanner. By scrutinising the advantages and disadvantages of each technique, summarised in section 6, the optimal workflow for the construction of virtual globe representations will become clear.

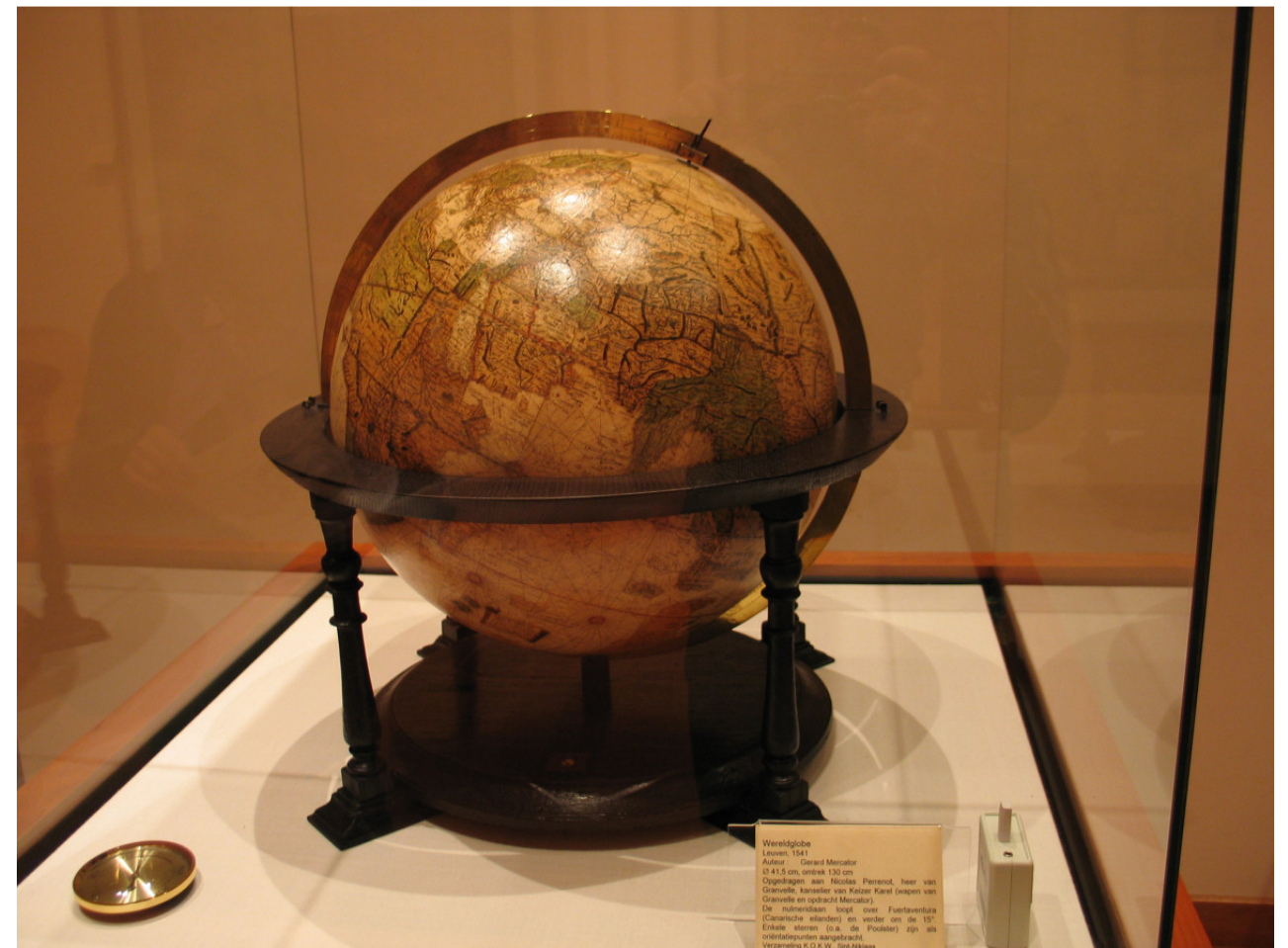

Figure 1: Terrestrial globe in the Mercator Museum, Sint-Niklaas, Belgium (source: Paul Hermans, www.wikipedia.org)

\section{Mercator Globes}

Gerardus Mercator (Gerard De Cremer, ${ }^{\circ} 1512$, Rupelmonde, Belgium; $\uparrow 1594$, Duisburg, Germany) is one of the most important $16^{\text {th }}$ century cartographers in the Low Countries. Even to this day global navigation makes use of his major achievement. His cylindrical map projection represents lines of constant course (i.e. rhumb lines or loxodromes) as straight segments (Nova et aucta orbis terrae descriptio ad usum navigantium emendate accommodata, 1569). Besides, Mercator created a large set of cartographic products. His map of Palestine (Amplissima terrae sanctae descriptio, 1537) still fits in the medieval tradition of maps with biblical interpretation. With the heart-shaped projection on his Orbis imago (1538), Mercator sought to represent the globe on a plane surface. In Duisburg he realised his map of Europe (1554) and most of his later maps. In 1578 he released Ptolemaeus' Geographia with the intention to correct the maps according to the author's mind (Watelet, 1994). The 
originally three independent volumes Galliae tabulae geographicae, Belgii inferioris geographicae tabulae and Germaniae tabulae geographicae were combined in Mercator's atlas and later completed by his son (Atlas sive cosmographicae meditationes de fabrica mundi et fabrica figura, 1585).

Although maps received ample treatment in his oeuvre, Mercator decided in around 1541 to prefer the construction of globes for commercial and financial reasons. Columbus' discovery of America enlarged political life with a lot of new territorial disputes. The Treaties of Tordesillas (1494) and Zaragoza (1529) defined a border meridian between Portuguese and Spanish foreign trade territories. Globes were especially suited for the representation of an overview of the whole world. They were excellent representations of territorial claims and were therefore favoured and patronised by the rulers. Both Gemma Frisius and Mercator dedicated their globes to Charles V or his entourage and in return received exclusive rights on the reproduction of their globes (Brotton, 2003). Mercator's terrestrial globe from 1541, the first globe dealt with in this paper, includes corrections on Gemma Frisius' globe (1535), while combining the antique worldviews of Ptolemaeus and Strabo with the works of Marco Polo and more of his contemporary cartographers such as Jacob Ziegler (1470-1549), Olaus Magnus (1490-1557) and Martin Waldseemüller (1470-1520). The result is a globe with a diameter of $41.5 \mathrm{~cm}$ of which the map image contains twelve north-south oriented ribbons. The second globe we will discuss is a celestial globe constructed in 1551 with approximately the same dimensions as the terrestrial globe (Blondeau, 1993). While the Discoveries changed the image of the world, the celestial representation remained largely based on Ptolemaeus' Almagest. But instead of the common ecliptic coordinates, Mercator used equatorial coordinates for the constellations of the stars. In spite of the differences, both the terrestrial- and celestial globes were sold together. That is probably why they have been mounted in a black-painted wooden construction with four bases and a circular frame along the ecliptic plane. Furthermore, a copper circular ring is mounted on this wooden frame with a longitudinal direction (Watelet, 1994).

\section{Image acquisition and pre-processing}

All images were taken with a Canon EOS 60D digital camera. However, the aperture, exposure time, film exposure and focal length varied during different acquisition steps, in order to acquire optimal images (Table 1). Both globes are varnished, which results in highly reflective areas on the surface of the globes. Therefore, all photographs were taken using a polarised filter. Furthermore, light sources around the globes needed to be controlled. In order to do so, a 'light tent' (www.lighttent.eu) was used to generate diffuse lighting and thereby avoid or minimise direct light beams on the globes. This 'light tent' has a cubed shape with an opening in one of the six sides. The other sides are covered with a white synthetic textile. A light spot is placed in the front left and front right side of the tent, accompanied with a frontal soft box. A synthetic textile sheet was placed in front of this soft box, to make this light even more diffuse. During the entire acquisition process of the images, the

configuration of the constant beaming lights and semi-opaque sheets was not changed. Only the rotation angle of the globes was altered during the acquisition of the images. The centres of all light beams were aligned with the ecliptic plane of the globe. This configuration is illustrated in figure 2 . 


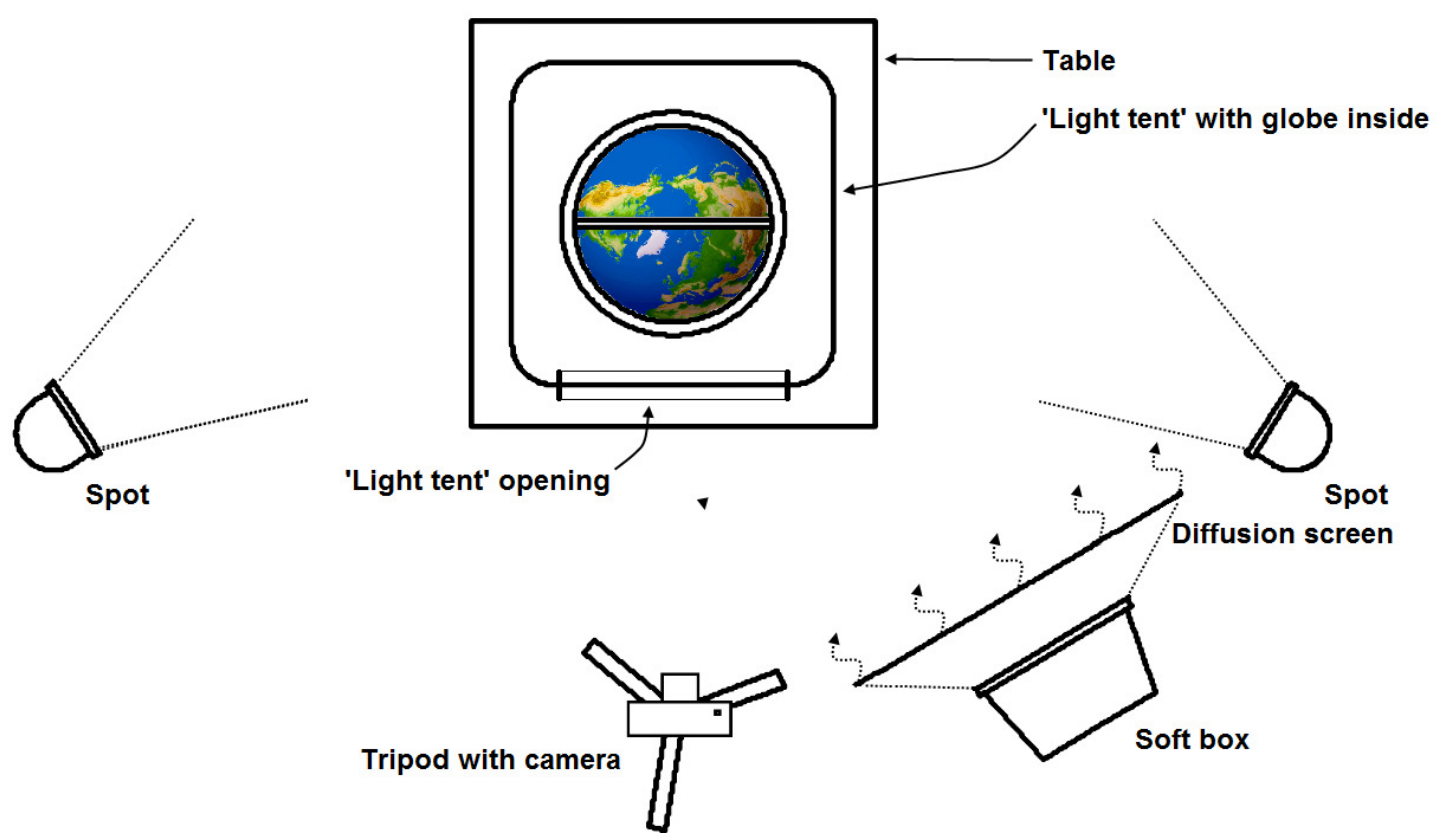

Figure 2: Configuration of the spots, camera and globe (Top view)

The Canon EOS 60D single lens reflex digital camera contains an 18 MP CMOS sensor, with a size of $22.3 \times 14.9 \mathrm{~mm}$ and each colour capturing cell has a size of $4.4 \mu \mathrm{m}$. To achieve an optimal overall sharpness of the images, each photograph of the ecliptic plane was focused on approximately $1 / 3$ of the sphere, north or south of the ecliptic plane. A tripod was used to keep a constant distance between the camera and the globe. Polar zones were photographed by hand, using variable camera parameters as a function of the illumination of the photographed area. These parameters are shown in table 1.

Table 1: Camera parameters during the image acquisition

\begin{tabular}{lcc}
\hline & Ecliptic plane & Polar area \\
\hline Lens & $50 / 1.8 \mathrm{~mm}$ & $35 / 2.0 \mathrm{~mm}$ and $24 / 2.8 \mathrm{~mm}$ \\
Aperture & $\mathrm{f} / 8$ & $\mathrm{f} / 5.6$ and $\mathrm{f} / 2.8$ \\
Film speed & ISO 200 & ISO 1600 \\
Exposure time & $0.6-1.0 \mathrm{sec}$ & $0.067-0.008 \mathrm{sec}$ \\
\hline
\end{tabular}

The lens used for the ecliptic plane images is included in a database of Adobe's Lightroom and Photoshop image processing software. Therefore a correction of the geometric aberration on all images of the ecliptic plane was possible (Ojanen, 1999). The images of the series of both the terrestrial and celestial globe contain a grey value scale and a standard GretagMacBeth colour checker (www.xrite.com). Based on the grey scale, the colour temperatures of the terrestrial and celestial globes were set to respectively $5100 \mathrm{Kelvin}$ and $5000 \mathrm{Kelvin}$, in order to get neutral grey values. The colour checker was used to set the colour balance correctly. On top of that, a set of filtering procedures was executed on all images to improve the contrast, sharpness and clarity of the images. The results of these procedures enable better image matching during the structure from motion modelling, as well as a better and sharper approximation of the real colours of the globes by the images. To compare the results of these procedures with the original images, a fragment of the celestial globe is shown in Figure 3. 

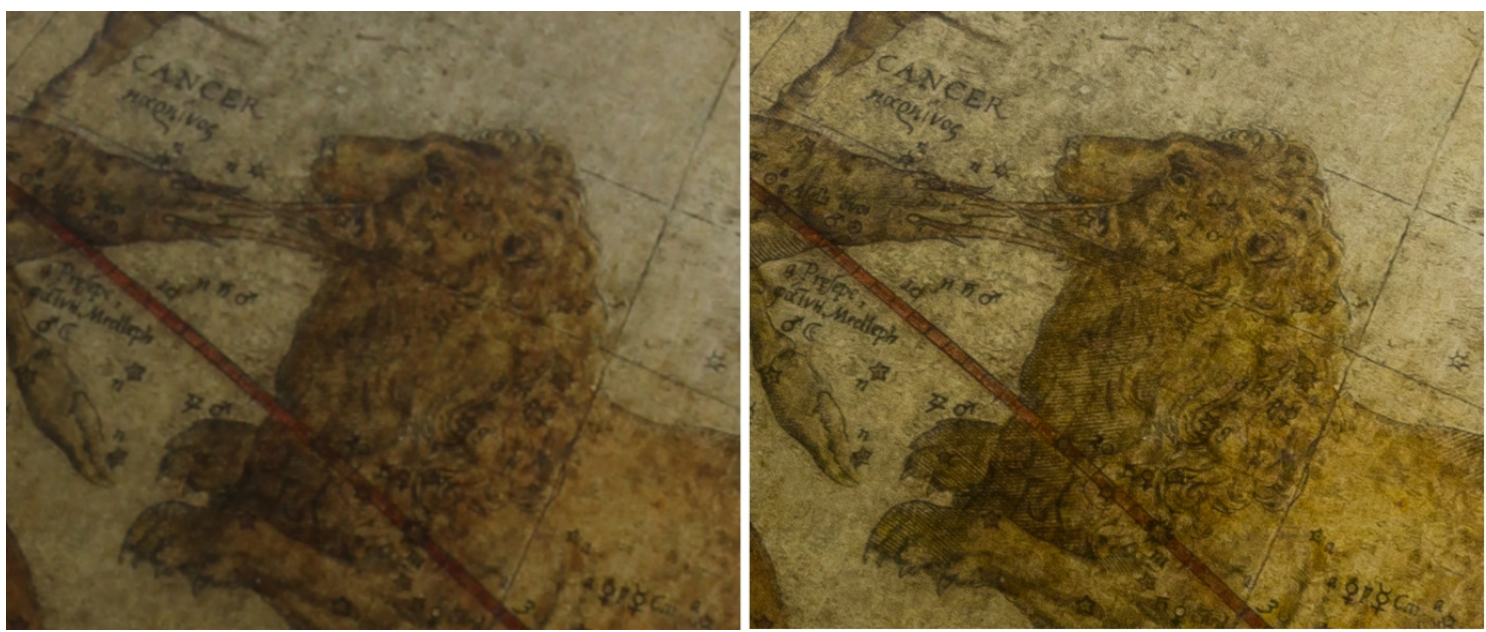

Figure 3: Fragment of the celestial globe before (left) and after (right) image correction

\section{Image Processing}

\subsection{Dynamic images}

For the generation of dynamic images, the Object2VR software package of Garden Gnome was used (www.gardengnomesoftware.com). This software package requires a series of images of the object and uses each image as a video frame for a movie of the object (Collmann, 2011). In this case, both the position of the camera and the orientation of the frame of the globe were kept static, in order to generate a single row moving image on the ecliptic plane. During the acquisition of the different images, the sphere of the globe itself was rotated with a step size of approximately $10^{\circ}$. After each rotation, an image of the globe was taken in the ecliptic plane. No supplementary images of the polar areas were included in this process. The resulting 36 images were organised chronologically and converted to either a QTVR or Flash file format. These formats can easily be embedded in an internet page using a Java script, calling the involved model file and appropriate viewer. Many interactive model viewers are available for this purpose. On the one hand, the possibility to generate animations in these formats is a big advantage when the model is placed on the internet as it increases the accessibility of the globe for the public. On the other hand, the generated files are quite large to download ( $85 \mathrm{Mb}$ for the QTVR and $82 \mathrm{Mb}$ for the Flash, with a frame size of 3456 by 5184 pixels), but these sizes could be reduced by using a lower resolution. However, there is no relation between the different images other than their sequence. This means that the resulting products do not contain real 3D information, although it visually looks like a 3D model, hence the term pseudo-3D model. A set of samples of the results are presented in figure 4 , demonstrating three neighbouring frames of a dynamic image.
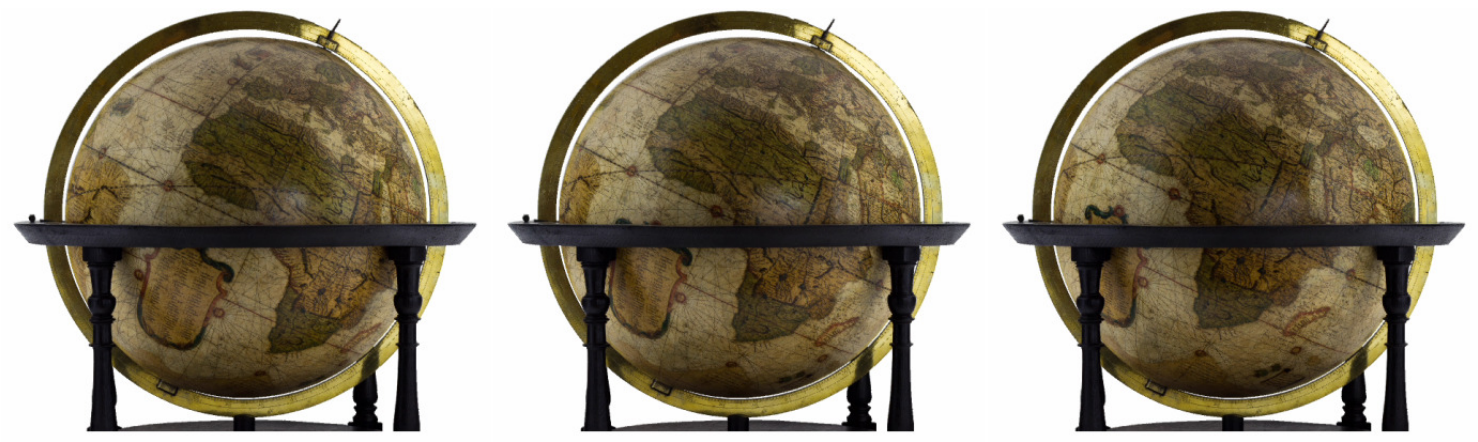

Figure 4: Screenshots of a dynamic image in a QuickTime environment

\subsection{Laser scanning and image draping}


3D scanning was used as modelling technique for globe representation, as a variant of the use of 3D range cameras (Adami, 2009). The technique is mainly applied in research about the real shape of the globe, e.g. aberrations from a hypothetic sphere. However, the results can be used for visualisation purposes as well, when the point set is coloured by image draping. A dense point set of the globes was acquired with a Leica HDS 6100 laser scanner. Using this phase-based terrestrial laser scanner (TLS), it is possible to acquire approximately 80 million points within 3.5 minutes. This type of scanner is very useful for applications in civil engineering (Nuttens et al., 2010) and cultural heritage (Stal et al., 2011), wherein high accuracy is indispensable and in which the distance between the scanner and the object to measure is confined. Corresponding with a mean distance between the scanner and the globes of 1.75 meter, an absolute single point accuracy of a few millimetres can be reached. The final data set contains an $(\mathrm{x}, \mathrm{y}, \mathrm{z})$-coordinate and an intensity value of the reflected signal for each point. The point set was acquired with a horizontal and vertical angular incremental $\alpha$ of $0.018^{\circ}$. With an average distance between the scanner and the globe of $1.75 \mathrm{~m}$, a minimum point spacing of $0.5 \mathrm{~mm}$ can be calculated $(s * \tan (\alpha))$.

The point set, acquired by the terrestrial laser scanner, can be combined with a photograph taken with a Canon EOS 60D DSLR camera in combination with a 50/1.8 mm lens. The acquisition of a single point set by TLS will result in occlusion zones. In order to have the same perspective geometry of the point set and the images, resulting in the same occlusion zones, both the point set and the image have to be acquired from the same position. In other words, the centre of the laser scanner and the optical centre of the camera - the so called 'no-parallax point' (NPP) - should coincide, as demonstrated in figure 5 (Littlefield (2006)). To determine the NPP of a specific camera and lens in relation to the centre of the laser scanner, two parameters have to be determined in the horizontal plane. The horizontal offset is shown in figure 5. As demonstrated in this figure, it will become clear that rotating the camera around the z-axis will keep the NNP on the same position.

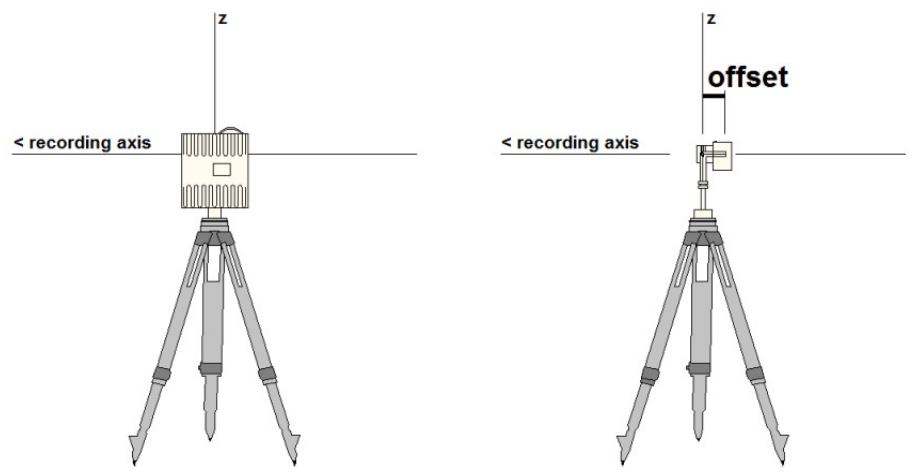

Figure 5: TLS set-up and corresponding camera offset

The placement of the NPP of a camera on the same location as the optical midpoint of the scanner can be obtained using the Nodal Ninja 3II camera bracket (figure 6). This bracket contains two measuring rods to perform an axis setup and is used for panoramic photographing as well (Lee et al., 2010). 

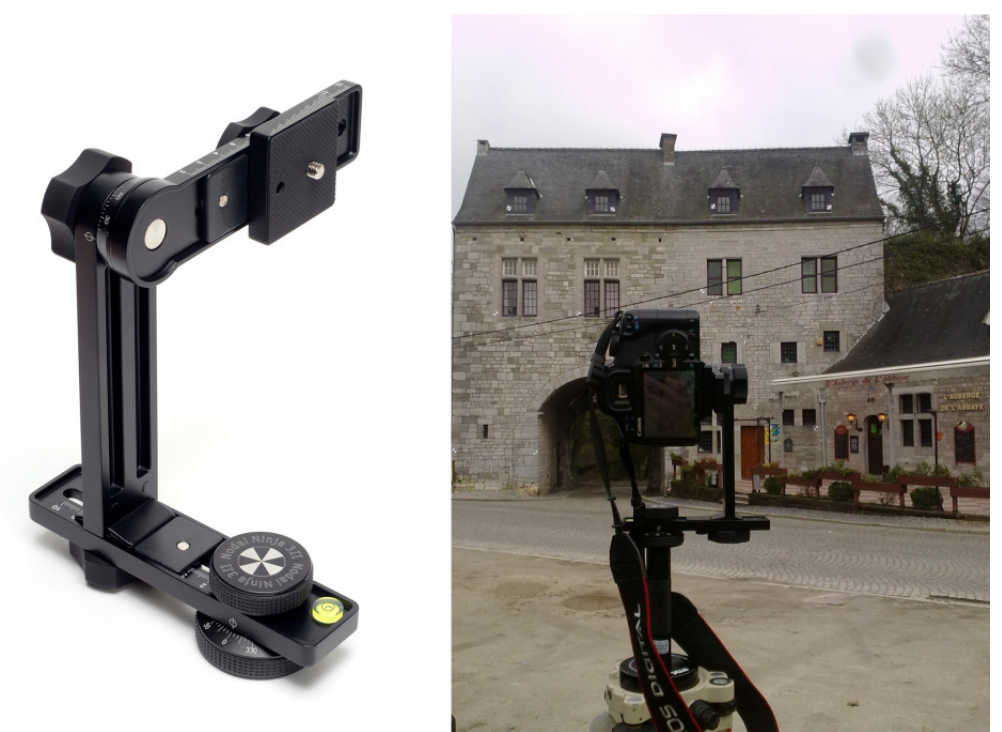

Figure 6: Camera bracket (Nodal Ninja 3 II, www.nodalninja.com)

The horizontal offset contains:

- $\quad$ The tripod mount length, which is camera dependent (L1) (figure 7 - left);

- The entrance pupil length, which is lens dependent (L2) (figure 7 - right).

In this case, a tripod mount length of $42 \mathrm{~mm}(\mathrm{~L} 1)$ and an entrance pupil length of $17.5 \mathrm{~mm}$ (L2) have been used for the combination of the Canon EOS 60D camera and the $50 \mathrm{~mm}$ lens. This resulted in a final horizontal offset of $38.5 \mathrm{~mm}$, but this value will differ for each camera and lens combination. The correct parameters can be calculated by manual offset determination, but online databases, like PanoTools Wiki (http://wiki.panotools.org, 2011), are available with these parameters for widely used camera and lens combinations.
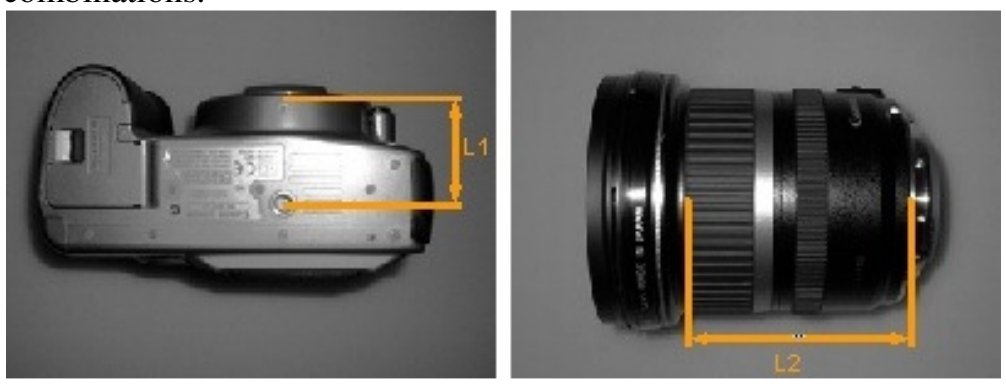

Figure 7: Tripod mount length (L1, left) and entrance pupil length (L2, right) (Source:

(http://wiki.panotools.org, 2011)

Texturing the point set is done by creating a set of corresponding points, which are points that are unambiguously recognisable in the point set and on the image (Nuttens et al., 2010). During the preparation of this campaign, nine circular black and white targets with a radius of $1.5 \mathrm{~cm}$ were glued on different places of the light tent and the frame of the globe as illustrated in figure 8 and figure 9 . During the target placement, an equal spread in the $\mathrm{x}-, \mathrm{y}$ - and z-direction was taken into account. Therefore, targets were placed on the copper ring (figure 8), the wooden frame and the back and front of the light tent (figure 9). Linking points in the point set with pixels in the images follows the same procedure as the registration of multiple point sets after a regular TLS campaign, where recognition of the targets is made possible by the big contrast of the intensity values of these targets.

The acquired point set is textured using Leica's Cyclone point processing software. The 'Texture Mapping' tool in this software package enables the draping of a photograph on a point set, using a minimum of 4 points for orthorectified photos and a minimum of 7 points for perspective photos. In 
both cases, the photo will be referenced on the point set, based on unambiguous matching points. These points will be selected in both the point set as on the image, as demonstrated in figure 8 .

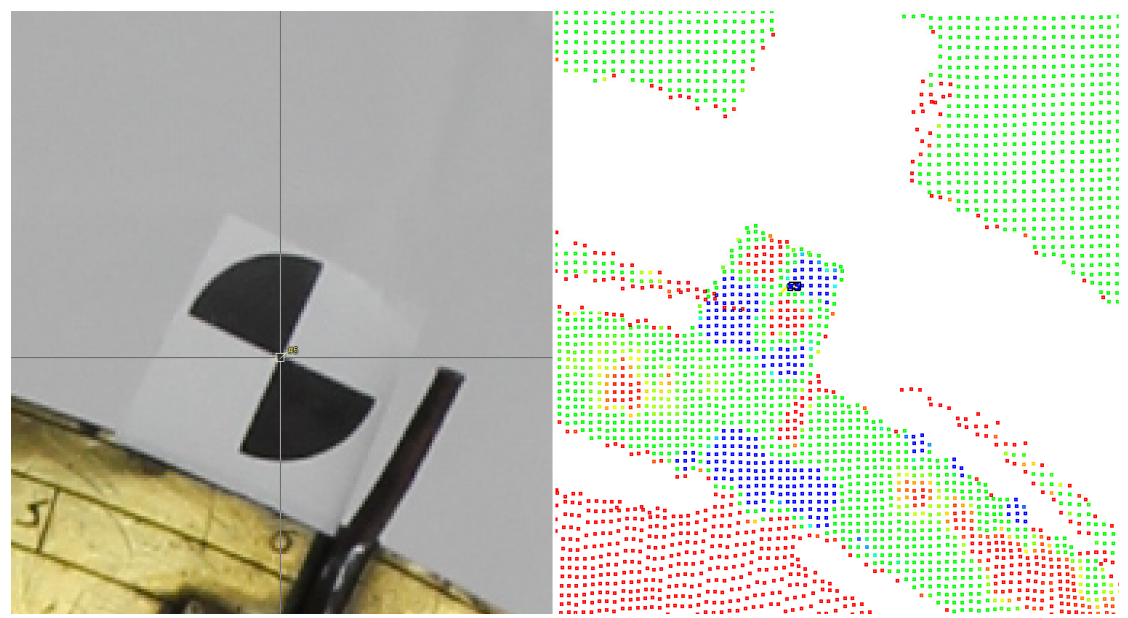

Figure 8: Registration point in the photo (left) and the point set (right)

The selected pairs of points are used to calculate the translation, rotation and scaling parameters, using a Direct Linear Transformation (Abdel-Aziz and Karara, 1971). The system of linear equations is solved in order to obtain the internal and external image parameters. Optionally, lens distortion parameters are determined using iterative collinear equations (Hu et al., 2008; Ming and Armenakis, 2010). User-defined threshold criteria are used by the algorithm, in order to accept or reject the result. These threshold criteria are based on the root mean square error (RMSE).

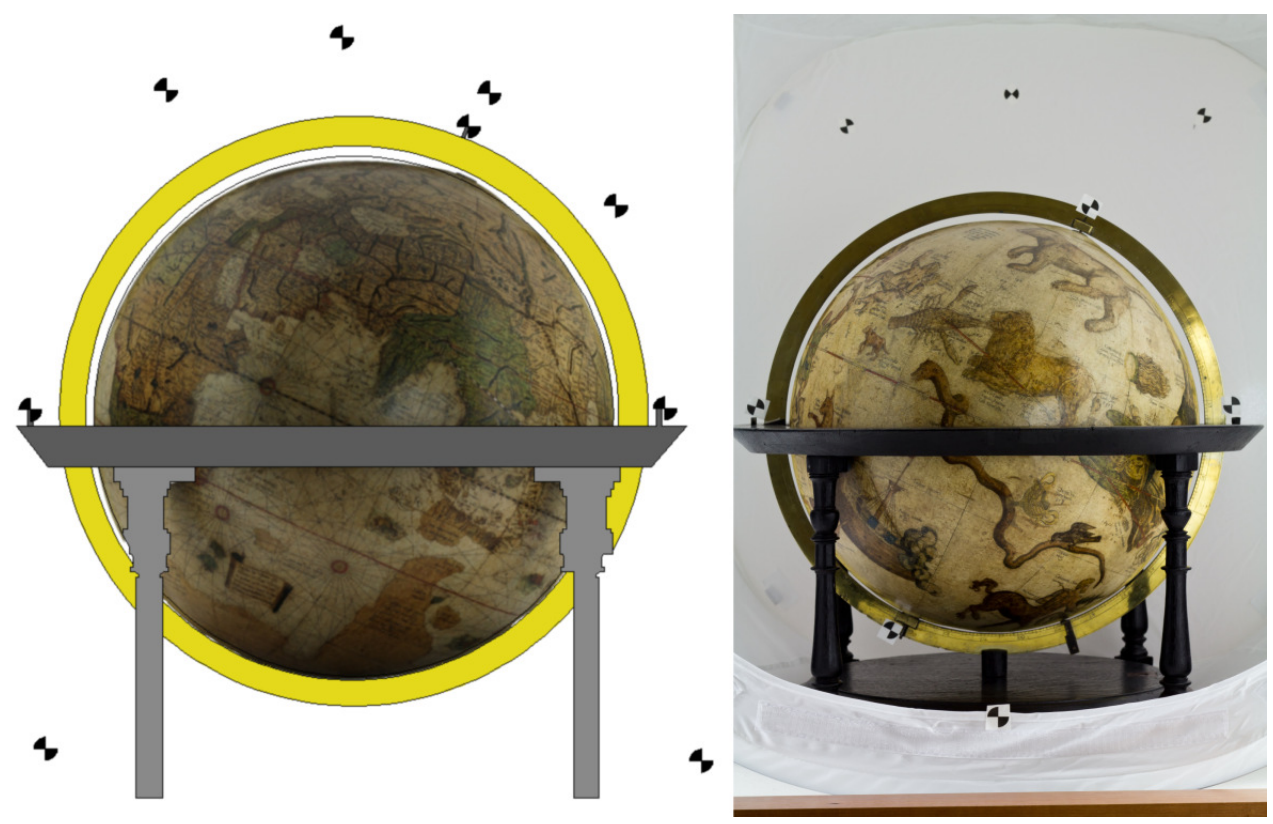

Figure 9: Target placement on the wooden frame, copper ring and the light tent

After linking all required targets in the point set and onto the images, the RMSE (Root Mean Square Error) value is calculated. If a registration is performed using the targets as unambiguous points, an RMSE value between 0.50 and 1.00 pixels can be obtained. The registration of the point set and the image, based on other recognisable features in the data, will result in a RMSE value of one pixel (Stal et al., 2011). The final result, after texturing, is a new point set, containing the measured $(\mathrm{x}, \mathrm{y}, \mathrm{z})-$ coordinate, the intensity of the reflected signal and the RGB-value of the corresponding pixel of a photo for each scanned point. Figure 10 demonstrates a screenshot of the textured point set based on 
the perspective photo. In the left figure, the intensity is visualised by a colour value, going from low (red) to high (blue). The right image demonstrates the textured point set with RGB-values.

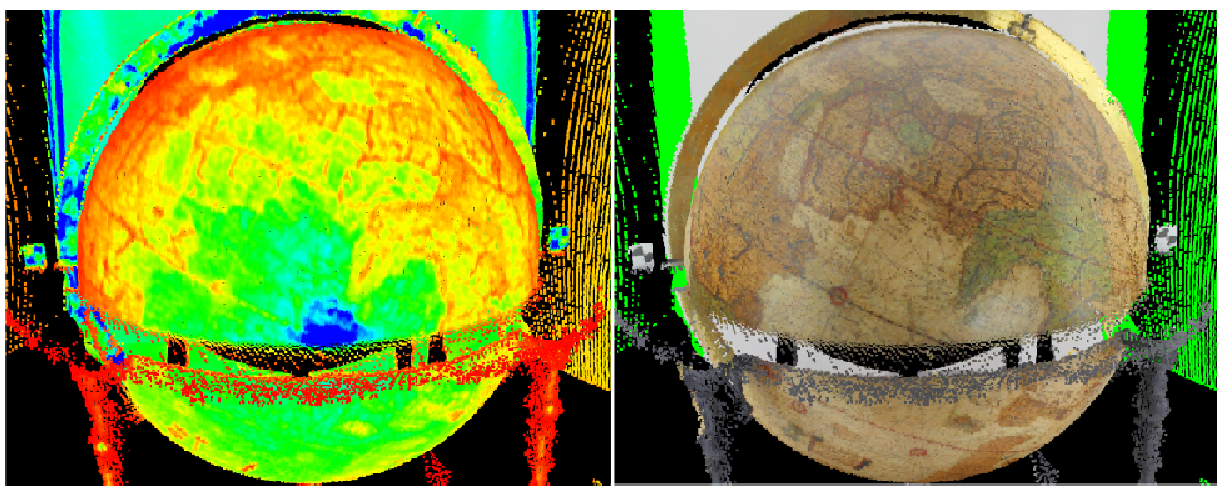

Figure 10: TLS point set with intensity (left) and RGB-values (right)

\subsection{Structure from motion}

Structure from motion is a technique in computer vision to acquire 3D geometry from 2D images (Pollefeys et al., 2000, Robertson and Cipola, 2009). Assuming a set of images, where each point of the study object is projected on a minimum of three images, the 3D positions of these points can be calculated by solving a system of geometrical matrices. If the geometric properties of an image are known, the use of two images is sufficient for the reconstruction of a 3D point. In most situations, an uncalibrated camera is used and the true position and orientation of the camera are unknown. In these cases, the projection of a 3D object on a 2D image plane and the inverse transformation of 2D image coordinates to 3D modelling object coordinates, requires the extrinsic and intrinsic camera parameters and the focal length (Robertson and Cipola, 2009). The intrinsic parameters and the focal length will be taken from the metadata of each image (EXchange Image Format, EXIF). The extrinsic transformation parameters will be calculated via the detection of matching points on the images. Different automatic matching techniques have been developed over the last decades (Chen et al., 1999; Heipke et al., 1992) and implemented in (commercial) software (Zhang et al., 1996). While digital photogrammetry strongly relies on the presence of camera calibration files for the orientation of the images, structure from motion software, like AutoDesk PhotoFly (www.labs.autodesk.com) or Agisoft PhotoScan (www.agisoft.ru), use series of images and the software automatically calculates the position of the camera, camera calibration parameters and relative scaling factors. This entire process can be split up into three steps (Verhoeven, 2011), which will be further discussed.

The alignment process consists of three steps. First, a set of characteristic points is automatically generated on each image. In the second step, these points are matched with characteristic points of other images. Finally, the scene structure is estimated based on this image matching. The result of this processing step is a $3 \mathrm{D}$ point set of matching points and a graphic representation of the image position. In preparation for the globe reconstruction, the globe's frame has been masked in the software and only the map-image is used for the alignment. In reality, the globe has been rotated during the acquisition of the photographs, but by masking the frame, the photographs appear to have been taken "around the globe". The software interprets this situation as if the globe were in a static situation and the images were taken around the globe, without presence of the wooden frame and copper ring.

Based on the aligned set of photographs, a 3D mesh is constructed. This mesh is calculated by reconstructing the local depths of each photograph. Based on the 3D matching points set, the local depth maps are merged into an overall 3D mesh. After this, the 3D mesh is refined by projecting pixels of different neighbouring images on the overall mesh. More information about the mathematical background of this modelling step is given by Robertson \& Cipola (2009) and Seitz et al. (2006). A screenshot of the result of the photo alignment and geometry building step is given in figure 11.

To texture the generated 3D mesh, all images need to be draped onto this mesh. Before starting this process, the dimensions of the texture images will be defined. In combination with the projection parameters of each image, the final texture map is blended. This blending process is performed by 
taking the maximum or minimum value of all corresponding pixels, or by taking the average of these pixel values.

After creating the textured 3D mesh and possible alignment of different sub-meshes (elliptic and polar meshes), the Agisoft software enables the export of the model to different file formats. Based on the requirements of the final product, the texture map can be ignored (e.g. export to Autodesk Exchange Format (dxf)) or included in the model (e.g. Wavefront object file (obj), Stanford polygon file (ply), Collada file (dae)). With these data formats, it is possible to assign a colour to each face in the mesh, or to generate a separate image file, containing the texture of the mesh. In general, the resolution of textures in separate files is higher than the assigned colours of faces.

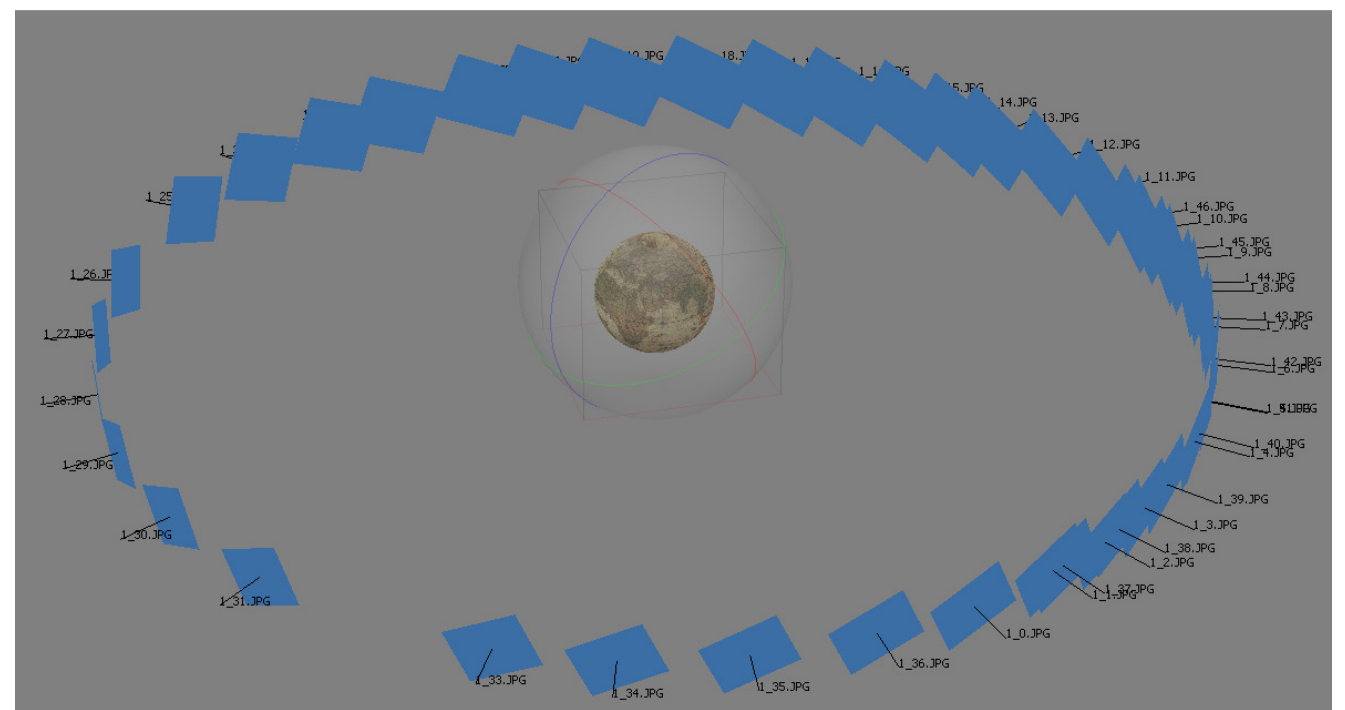

Figure 11: Reconstruction of the camera positions and 3D representation of the terrestrial globe

\subsection{Georeferencing and merging fragments}

The last technique with which to make a 3D model of the globe is by georeferencing fragments of images of the globe (figure 12), as presented by Dorffner (1996) and Hruby et al. (2005, 2006). An absolute coordinate system will be used in order to generate a virtual representation. It is thus important to discuss some theory of map projections, in order to be able to predict and describe distortions (Gede, 2009a). During the acquisition and processing of the images of the globes, these globes are assumed to be spherical and the centre of this sphere is supposed to be on the central optical axis of the camera. According to these properties, the globe is projected on the camera sensor, using the vertical perspective projection (Snyder, 1987). If the centre of the sphere and the optical axis do not coincide, a more complex tiled perspective projection is used. Next to the focal length of the camera, the distance between the camera and the globe determines the position of the horizon and the size of the error on the (pseudo) parallels. The area, length and angular errors on the (pseudo) meridians and (pseudo) parallels will increase for increasing distances from the centre of projection.

As a result, it is essential to take map fragments from the images as near to the centre of the projection as possible. The terrestrial globe has a grid painted on it, with meridians each $15^{\circ}$ and parallels each $10^{\circ}$. This grid is digitalised in a GIS (Geographic Information System) and the resulting grid is georeferenced within a world-wide reference system. The image fragments were chosen from different images, but only one single central fragment was chosen from one single image. To cover the entire earth's surface, a total of 432 fragments were selected, and the corners of each fragment were linked to the digital grid. 


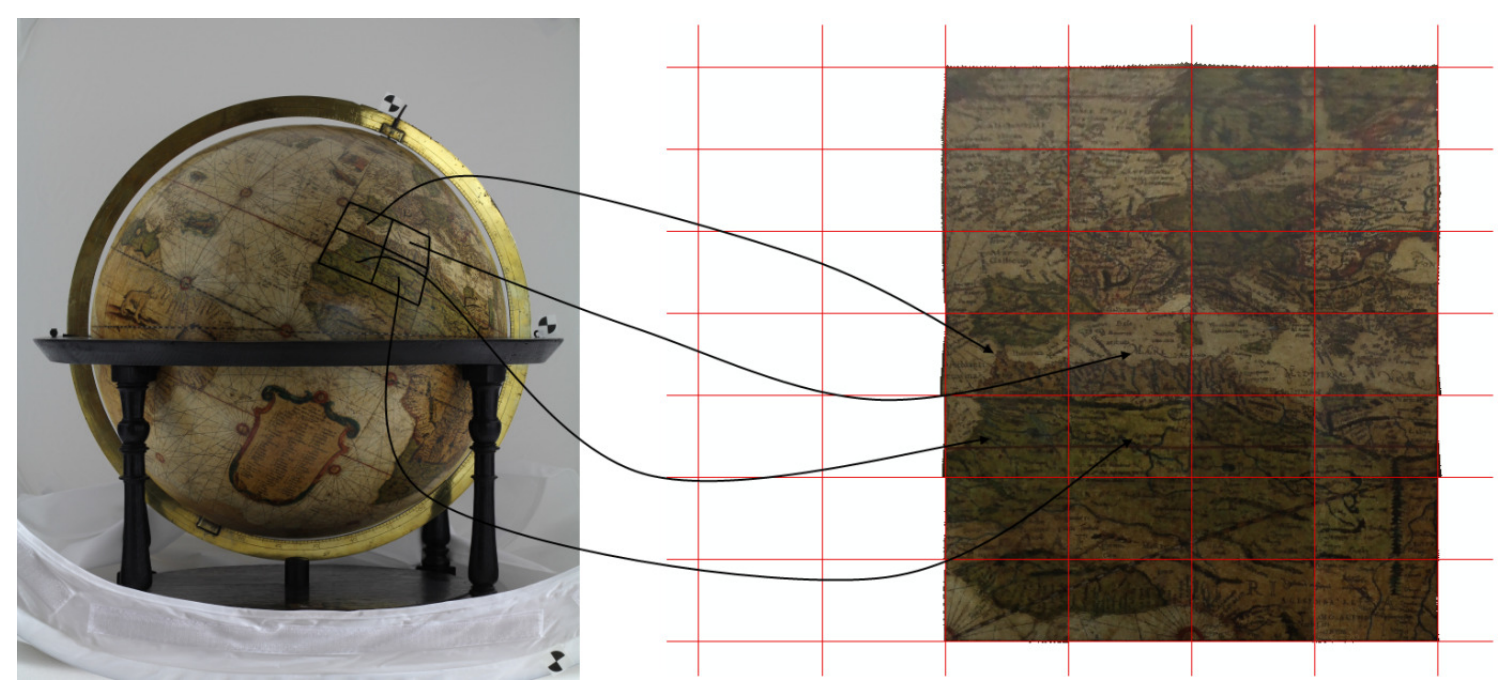

Figure 12: Reconstruction of the globe by georeferencing

The technique of generating 3D models by georeferencing fragments of the images has some drawbacks. First of all, the procedure has a relatively time consuming workflow. To cover the entire earth's surface, 432 fragments have to be cut from a selected photograph. After this, all four corners of the 432 fragments have to be aligned with the digital grid. Another drawback is the fact that only four points on the edges of a fragment will be used as unambiguous reference points. In the centre of a cell, the deformations will be maximal and the error vector points to the centre of the globe. This is caused by the non-spherical interpolation of the fragments, and the flat projection of a flat image on a small part of the sphere. Moreover, erroneous geographic placement of objects on the map of the globe in relation to the used grid will result in geometric displacements as well (Hruby et al., 2006). This displacement will occur in all discussed methods in this article, but with the explicit use of an absolute reference system for the model, and a grid with a user defined resolution, the error will occur most prominently.

\section{Visualising the results}

The results of this research can be consulted in different commercial and non-commercial viewers. A very useful software package for 3D mesh visualisation, analysis and modification is MeshLab (www.meshlab.sourceforge.net). All file types mentioned in this article can be handled in this open source software, along with many other file types. For the visualisation of wrl files in an internet browser, Cortona 3D (www.cortona3d.com) could be used, but we experienced better performance with stand-alone software. The consultation of the dynamic images in a browser requires a Flash- or QuickTime plug-in. The digital models of the Mercator globes will be presented within the framework of the 'Mercator Digital' exhibition (www.kokw.be).

\section{Discussion}

The generation of 3D models of globes is a challenging task and the presented approaches all have different advantages and drawbacks, depending on the desired quality, processing speed and more importantly: the purpose of the modelling. Within the context of the case study used in this article, the main purpose of the 3D models was to present the globes to the public during the Mercator exposition. The first discussed technique (dynamic imaging) is useful for both visualisation purposes and the research on the cartographic content of the globes. During the reconstruction of the globe, which is solely based on images, no further deformations occurred after the acquisition of the images. Actually, the result can be seen as a dynamic database, enabling fast scrolling through a set of original high resolution images. However, neither 3D visualisation, nor geometrical analysis can be performed with these results, but the results are easily publishable on the internet. This in contrast to the $3 \mathrm{D}$ scanning 
technique, whereby deformation measurements of the globes are possible, as discussed in Adami (2009). In the configuration employed using the light tent, a full coverage by the scanner was not possible, since the main purpose of this project was visualisation and the quality of the images was not satisfactory outside this light tent. A full coverage would require targets around the globe and a free field of view. However, a 3D analysis would still be possible for the measured part of the globe, but the image draping technique, as discussed in this article, does not seem to be suitable for visualisation purposes of detailed textures. The georeferencing of images or image parts is the most common technique for globe visualisation in literature. The method of Dorffner (1996) was used to perform the referencing of the images in a GIS based environment. The method is very straightforward on the one hand, but time consuming on the other hand. Since no sphere was used but rather a rectangular grid as a framework for the georeferencing, the number of images had to be substantial in order to keep the geometrical deformation limited.

Based on the results of this article, the most promising technique for 3D modelling of the globes is structure from motion. Although the processing of the images requires a high performance computer, the results are satisfactory from both a visualisation and a geometric point of view. Before the final presentation of the structure from motion results, the models required some post-processing near the North pole and South pole. Both the terrestrial- and the celestial globe will be presented in 3D during the Mercator-exhibition.

\section{Conclusion}

In this article, an overview has been provided of modelling techniques for the virtual representation of two historical globes, focussing on visualisation purposes. Images of two historical globes, constructed by Mercator, are taken under studio conditions and pre-processed in order to have an improved approximation of the real colours of the globes by the images. Thereafter, the images are used for different processing techniques. It has become apparent that all four techniques - dynamic imaging, laser scanning, structure from motion and image georeferencing - have different advantages and drawbacks. After analysing and performing all four techniques, the following statements are formulated in table 2. The discussed methods using terrestrial laser scanning and manual georeferencing are not very suitable for 3D globe representation in this context. The laser scanning method can be used for geometric analysis rather than texture analysis of the globes, but this is beyond the scope of this paper. The results generated by georeferencing are acquired in a time consuming process and are of much lower quality than the results generated by dynamic imaging and structure from motion.

Table 2: Advantages and disadvantages of the used reconstruction techniques

\section{Dynamic imaging (Obj2VR)}

Advantage

Easy to use and no high performance hardware required

Good exchangeability and accessibility via the internet

\begin{tabular}{|c|c|}
\hline Good exchangeability and accessibility via the internet & Geometric quality analysis impossible \\
\hline \multicolumn{2}{|c|}{ Terrestrial laser scanning and image draping (Leica HDS 6100 and Leica Cyclone) } \\
\hline Advantage & Drawback \\
\hline High accuracy geometric model & Expensive acquisition hardware \\
\hline Quality of point set independent of environmental light sources & $\begin{array}{l}\text { Full coverage of both laser scanner and camera not } \\
\text { possible }\end{array}$ \\
\hline \multicolumn{2}{|c|}{ Structure from motion (Agisoft PhotoScan) } \\
\hline Advantage & Drawback \\
\hline Straightforward 3D model generation & High performance computer required \\
\hline Flexibility in resulting digital formats & Black-box processing \\
\hline \multicolumn{2}{|c|}{ Georeferencing and merging images (ESRI ArcGIS) } \\
\hline Advantage & Drawback \\
\hline User controlled processing & Time consuming \\
\hline & High geometric distortion in the centre of unit cells \\
\hline
\end{tabular}

\section{Drawback}

No true $3 \mathrm{D}$ results

Geometric quality analysis impossible
High geometric distortion in the centre of unit cells 
Based on these statements and experiences, we conclude that for the particular case of mid-sized historical globes, the dynamic imaging and photogrammetric modelling (in this case: structure from motion modelling) techniques are the most suitable for the generation of virtual globes aimed at public exhibitions. The results from the dynamic imaging are very useful for further semantic and historical research of the globes. Globe details, like compass cards, cartouches and text in general, are clearly represented in the model. The resolution of the original images is retained and the constant angular increment of the globe still results in a 3D impression. Some of the image resolution will be lost by structure from motion processing, but this reduction is mainly manageable by using the correct processing parameters. However, the improvement of the geometric accuracy of the products from the structure from motion technique requires further research. Nevertheless, this 3D modelling technique already offers very satisfactory visual results.

\section{Acknowledgement}

The authors would like to express their gratitude to the 'Koninklijke Oudheidkundige Kring van het Land van Waas' (KOKW) for their permission and opportunity to take images of the two historical globes and the ability to present the results of this research during the Mercator's exposition.

\section{Biographic notes}

Cornelis Stal ( ${ }^{\circ} 1985$, Waalre, the Netherlands) is a $\mathrm{PhD}$ student working on the combination of airborne and terrestrial laser scanning for 3D city modeling. His special interest is in the (automatic) generation of geometric, radiometric and semantic rich 3D models, derived from irregular point sets and other spatial datasets. This means that both laser scanning as a discipline in the land survey and geo-IT (GI-systems, GI-programming, GI-management,...) are important pillars of his research.

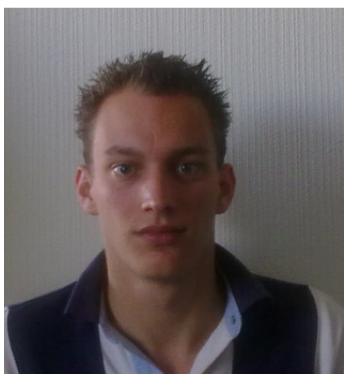

References

Abdel-Aziz, Y. and Karara, H. (1971). 'Direct Linear Transformation into Object Space Coordinates in Close-range Photogrammetry', Close-range Photogrammetry, Urbana, Illinois, USA, pp. 1-18.

Adami, A. (2009). 'From Real to Virtual Globe: New Technologies for Digital Cartographic Representation', e-Perimetron, 4, pp. 144-160.

Blondeau, R. (1993). Mercator van Rupelmonde, Lannoo, Tielt, Belgium.

Brotton, J. (2003). Trading Territories Mapping the Early Modern World, The University of Chicago Press, Chicaco, Il, USA.

Chen, L., Lo, C., Liu, C. and Chen, A. (1999). 'Orientation Modeling by Matching Image Templates of a GCP Database', XXVIIIth Asian Conference on Remote Sensing, Kuala Lumpur, Malaysia, p. 6 (on CD-ROM).

Collmann, R. (2011). 'Developments in Virtual 3D Imaging of Cultural Artefacts'. Ariadne, 66, p. 10 (on CD-ROM).

Dorffner, L. (1996). 'Der digitale Behaim-Globus - Visualisierung und Vermessung des historisch wertvollen Originals'. Cartographica Helvetica, 14, pp. 20-24. 
Gede, M. (2009a). 'The Projection Aspects of Digitising Globes', XXIVth International Cartographic Conference, Santiago, Chile, p. 10 (on CD-ROM).

Gede, M. (2009b). 'Publishing Globes on the Internet'. Acta Geodetica Geographica Hungaria, 44, pp. 114-148.

Heipke, C., Kornus, W., Strunz, G., Thiemann, R. and Colomina, I. (1992). 'Automatic Photogrammetric Processing of Spot Imagery for Point Determination, DTM Generation and Orthoprojection', International Archives of Photogrammetry and Remote Sensing, 29, pp. 465471.

Hruby, F., Plank, I. and Riedl, A. (2005). 'Potential of Virtual 3D-Facsimiles - Exemplified by the Earth Globe of Gerard Mercator (1541)', 22nd ICA Cartographic Conference, La Coruña, Spain, p. 9 (on CD-ROM).

Hruby, F., Plank, I. and Riedl, A. (2006). 'Cartographic Heritage as Shared Experience in Virtual Space: a Digital Representation of the Earth Globe of Gerard Mercator (1541)'. e-Perimetron, 1, pp. $88-98$.

http://wiki.panotools.org, 2011. PanoTools Wiki (Accessed 24 March 2011).

Hu, C., Wang, Y. and Yu, W. (2008). 'Mapping Digital Image Texture onto 3D Model from LiDAR data'. International Archives of Photogrammetry, Remote Sensing and Spatial Information Sciences, 37, pp. 611-614.

Lee, H., Tateyama, Y. and Ogi, T. (2010). 'Realistic Visual Environments for Immersive Projection Display System', 16th International Conference on Virtual Systems and Multimedia (VSMM), Seoul, Korea, pp. 128-132.

Littlefield, R. (2006). 'Theory of the No-Parallax Points', www.janrik.net/PanoPostings/NoParallaxPoint/TheoryOfTheNoParallaxPoint.pdf (Accessed 23 March 2011).

Ming, J. and Armenakis, C. (2010). 'Fusion of Optical and Terrestrial Laser Scanner Data'. International Archives of Photogrammetry, Remote Sensing and Spatial Information Sciences, 38, pp. 156-161.

Nuttens, T., De Wulf, A., Bral, L., De Wit, B., Carlier, L., De Ryck, M., Stal, C., Constales, D. and De Backer, H. (2010). 'High Resolution Terrestrial Laser Scanning for Tunnel Deformation Measurements', The XXIV FIG International Congress 2010, Sidney, Australia, p. 10 (on CD ROM).

Ojanen, H. (1999). 'Automatic Correction of Lens Distortion by Using Digital Image Processing', Technical Report, Rutgers University, New Brunswick, NJ, USA, p. 5 (on CD-ROM).

Pollefeys, M., Koch, R., Vergauwen, M. And Van Gool, L. (2000). 'Automated Reconstruction of 3D Scenes from Sequences of Images', ISPRS Journal of Photogrammetry and Remote Sensing, 55, pp. 251-267.

Robertson, D. and Cipola, R. (2009). 'Structure from Motion', in: Practical Image Processing and Computer Vision, ed. by Varga, M., John Wiley, Hoboken, NJ, USA, pp. 23. 
Seitz, S., Curless, B., Diebel, J., Scharstein, D. and Szeliski, R. (2006). 'A Comparison and Evaluation of Multi-view Stereo Reconstruction Algorithms', IEEE Computer Society Conference on Computer Vision and Pattern Recognition, Washinton, USA, pp. 519-526.

Snyder, J. (1987). ‘General Perspective Projection', in: Map Projections - A Working Manual, ed. By Snyder, J., USGS Professional Paper, Washington, USA, pp. 169-181.

Stal, C., De Wulf, A., Nuttens, T., De Maeyer, P. and Goossens, R. (2011). 'Reconstruction of a Midieval Wall: Photogrammetric Mapping and Quality Analysis by Terrestrial Laser Scanning', 31th EARSeL Symposium, Prague, Czech Republic, p. 12 (on CD-ROM).

Verhoeven, G., (2011). 'Taking Computer Vision Aloft - Archaeological Three-dimentional Reconstruction from Aerial Photographs with PhotoScan'. Archaeological Prospection, 18, pp. 6773.

Watelet, M. (1994), Gerardus Mercator Rupelmundanus, Mercatorfonds, Antwerpen, Belgium.

Zhang, J., Zhang, Z., Shen, W. and Wang, Z. (1996). 'VirtuoZo Digital Photogrammetry System: its Theoretical Foundation and Key Algorithms'. International Archives of Photogrammetry and Remote Sensing, 31, pp. 424-429. 